\title{
ESTIMACIÓN DEL IMPACTO PAISAJÍSTICO DE LAS CARRETERAS
}

\author{
(IMPACT ASSESSMENT OF ROADS ON LANDSCAPE)
}

Ignacio Cañas Guerrero, Ingeniero Agrónomo

E.T.S.I. Agrónomos de Lugo)

ESPAÑA
Fecha de recepción: 27 - IV - 93

$113-18$

\section{RESUMEN}

Quizá uno de los medios más importantes por los cuales la opinión priblica conoce el paisaje sea a través de la carretera. En España tenemos más de $325.000 \mathrm{~km}$ de carreteras que son utilizadas por 16,5 millones de vehículos, su interés es pues grande. Esta importancia parece que en los próximos años seguirá creciendo. Este proceso va unido a una sensibilidad cada vez mayor por parte de la población con los temas medioambientales. Esto da lugar a que nos encontremos con enfrentamientos crecientes entre el desarrollo y la conservación de la naturaleza.

Estos conflictos pensamos que pueden reducirse, si ya en las primeras etapas de la planificación y diseño se identifican las áreas más problemáticas y sus posibles soluciones. Dentro de la relación carretera-medioambiente la importancia de los estudios paisajisticos es manifiesta, ya que uno de los aspectos que la opinión príblica valora de forma mas directa es la integración de la carretera en el paisaje.

Precisamente el objetivo que pretendemos con este trabajo es ver cuáles son las principales etapas en el análisis del tema paisajistico en los proyectos de carreteras. No se trata de una descripción exhaustiva sino de un comentario sobre las diversos pasos de la estimación del impacto paisajístico que nos pueda servir de marco para su realización en diferentes proyectos.

\section{SUMMARY}

Perhaps one of the most important ways in which public opinion knows the landscape is through roads. The $325,000 \mathrm{~km}$ of roads in Spain are of great importance since they are used by 16.5 million vehicles. It seems that this importance will continue to grow in the years to come. This process goes together with the increasing sensitivity of the population where environmental issues are concerned. For this reason we constantly face confrontations between the developement and the nature preservation.

We believe it possible to reduce these conflicts by identifying the most problematic areas and the possible solutions in the initial stages of planning and design. In the road - landscape relation the importance of landscape studies is obvious since one of the aspects the public opinion values most directly is the integration of the road in the landscape.

The aim of this paper is precisely to identify the principal stages in the analysis of the landscape issue in road projects. This is not a thorough description but a comment of various steps to be taken in the estimation of the impact on landscape which can serve as the starting point to be developed further in different projects.

\section{I.-INTRODUCCIÓN}

Al estudiar la relación entre la carretera y el paisaje se observa que ésta puede enfocarse desde dos puntos de vista: $1^{\circ}$ ) paisaje que se ve desde la carretera; 2 ) paisaje desde donde se ve la carretera. Algunos colectivos suelen hacer especial hincapié sólo en la segunda, pero es necesario remarcar que una gran parte de la visualización de los recursos paisajísticos se tiene desde las carreteras. Por ello el enfoque de la integración de la carretera en el paisaje no debe limitarse sólo a una postura defensiva; sino a enriquecer la experiencia visual fe los que la utilicen persiguiendo (como indica Poter R. D. and Wagar J.A. 1971) entre otros los siguientes objetivos: 
$1^{\circ}$ Ilustrar y guiar la orientación del conductor al paisaje ofreciendo un cuadro bien estructurado, distintivo y lo mas variado posible, donde él se pueda localizar a sí mismo, localizar el sistema de transporte y los mayores rasgos del paisaje para reconocerlos y sentir cómo se acerca y se mueve entre ellos.

$2^{2}$ Ayudar al observador a profundizar en el significado de su entorno y en el entendimiento de la historia y naturaleza del paisaje adyacente.

\section{2.-ETAPAS EN EL PROCESO DEL ESTUDIO DEL IMPACTO PAISAJÍSTICO}

Aun remarcando que las consideraciones paisajisticas pueden ser muy importantes, es necesario elegir el método adecuado para cada proyecto evitando un estudio muy profundo si el efecto es pequeño o moderado.

En la estimación del impacto se distinguen dos elementos: los recursos paisajísticos y los observadores. En la gran mayoría de los estudios se suele hablar tan sólo del impacto visual ( USDOT 1981, Shanahan D. et al 1986). No obstante es evidente que los efectos de la carretera no son sólo visuales sino también auditivos, e incluso olfativos. Aunque normalmente sean los impactos visuales los mas significativos.

Dentro del análisis del impacto paisajístico de las carreteras, podemos distinguir la valoración del paisaje y la valoración de la carretera. Con la primera se trata de estimar la calidad de éste. Con la segunda se intenta ver, en un primer nivel, si el diseño expresa las funciones del proyecto o si los detalles visuales son congruentes entre ellos y con el conjunto total; en un segundo nivel se trata de valorar su adaptación al paisaje, si contrasta fuertemente o si tapa vistas.

En el estudio de los observadores distinguimos: facilidad de apreciarse, número de observadores e interés de los mismos. En el esquema aparece un estudio paisajístico de una carretera.

Como se aprecia el proceso consiste en valorar el paisaje, en valorar la vía en sí misma y sobre todo en relación con el paisaje para ver su adaptación a éste. Paralelamente se estima la respuesta del observador, que, junto con la adaptación de la carretera al paisaje, nos dará el impacto paisajístico. A continuación

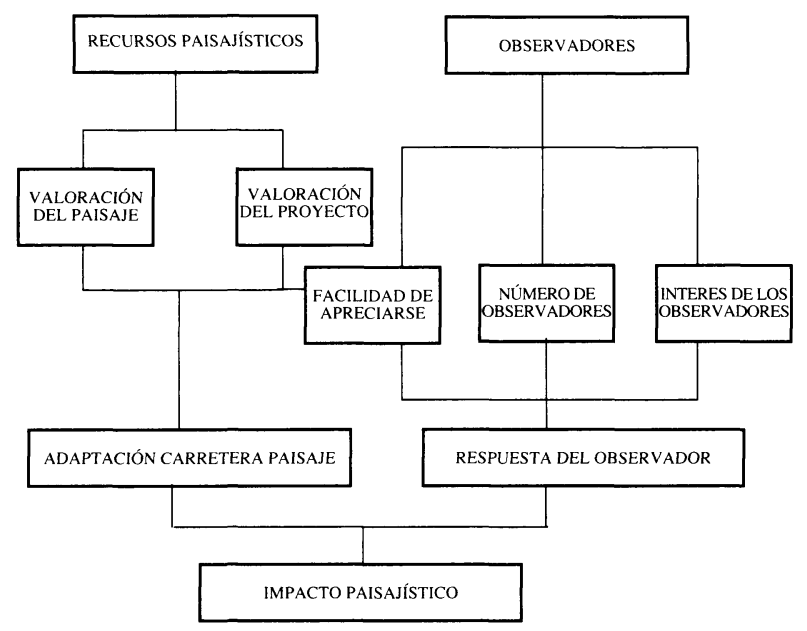

Esquema del estudio paisajístico de una carretera

aparece un resumen de las fases de la evaluación del impacto paisajístico de una carretera, que se desarrolla en el apartado siguiente.

\section{A - VALORACIÓN DEL PAISAJE}

1. Estudio de los paisajes regionales.

2. Identificación de las vistas claves: elección de los puntos de observación y valoración a lo largo de la carretera.

3. Valoración y delimitación de las unidades paisajísticas.

\section{B- VALORACIÓN DEL PROYECTO}

\section{C- OBSERVADORES}

1. Facilidad de apreciarse.

2. Número de observadores.

3. Interés de los observadores.

D- ESTIMACIÓN DEL IMPACTO

\subsection{Valoración del paisaje}

\subsubsection{Estudio de los paisajes regionales}

El estudio de los paisajes regionales puede ayudarnos a establecer el marco de referencia para comparar los efectos visuales de las distintas alternativas y 
determinar el significado de estos efectos sobre una unidad superior.

En otras palabras "no podemos estimar los efectos visuales de un proyecto hasta que no entendamos cómo el entorno inmediato visual se relaciona con el entorno de la región geográfica" ( USDOT 1981 ).

Los principales elementos que componen el estudio de los paisajes regionales son:

- Unidades fisiográficas.

- Vegetación.

- Modificaciones antrópicas.

Tanto las unidades fisiográficas como la vegetación suelen establecerse a partir de sus respectivos mapas, simplificando y utilizando los aspectos que más directamente influyen en el paisaje: así la vegetación se puede agrupar para obtener áreas homogéneas desde el punto de vista paisajístico en las siguientes: cultivos de regadío, cultivos de secano, pastizales, matorrales, mosaicos con formaciones de matorral y arbóreos, frondosas de hoja persistente, frondosas de hoja caduca, pinares y formaciones en galería.

Por lo que se refiere a las modificaciones antrópicas se puede clasificar en: zonas afectadas por la expansión urbana, zonas afectadas por las actividades extractivas, zonas afectadas por la red de transportes (aeropuertos, autopistas y autovías, carreteras y ferrocarriles) y embalses. En el mapa $n^{2} 1$ aparecen los paisajes regionales de la Comunidad Autónoma de Madrid (Cañas, 1992), que puede ilustrar este proceso.

Aunque no es necesario que aparezcan en el plano de paisajes regionales, es conveniente recoger en un plano las diversas zonas protegidas por su interés ecológico (parques naturales, parajes naturales, etc.) o aquellas zonas que aunque no estén declaradas oficialmente están solicitadas o propuestas por diversos colectivos para incluirse en zonas de protección. Es recomendable también señalar las zonas de interés histórico o arqueológico.

\subsubsection{Identificación de vistas claves y elección de los puntos de observación}

Se trata en este apartado de establecer los puntos de observación para valorar los recursos paisajísticos. La forma de elegir éstos depende del sistema de evalua- ción que se vaya a seguir. El más sencillo es escoger aquellos que son mas representativos; bien por el número de observadores, por ejemplo a la salida de una población, o bien por ser característico de ese tipo de paisaje.

Hay autores que estudian (Potter R.D. and Wagar J.A. 1971) entre otras una evaluación cada $350 \mathrm{~m}$ de carretera, de la que se tomaría una serie de fotografías. Como es evidente en la mayoría de los casos, el costo es alto y además esto podría dar lugar a un exceso de información no relevante.

\subsubsection{Valoración y delimitación de las unidades pai- sajísticas}

En este apartado se valora propiamente los recursos paisajísticos. Podemos distinguir dos subetapas en el proceso de valoración:

- Inventario de los recursos.

- Evaluación de los recursos.

El inventario lo podemos hacer principalmente de tres formas: gráficamente, narrativamente, lista de chequeo.

Los inventarios gráficos registran rasgos individuales que pueden ser muy útiles para el diseño pero dificultan la comparación de alternativas.

Los inventarios narrativos suelen ser útiles si son pocas unidades, además la comparación entre alternativas no es fácil.

Los inventarios en forma de lista de chequeo facilitan la comparación entre diversas alternativas e incluso entre diversos puntos de observación, aunque necesitan un amplio texto explicativo.

Los inventarios los podemos expresar en forma de cantidades físicas o en términos relativos como bajo, moderado o alto.

A la hora de la evaluación de los recursos hay una amplia variedad de métodos; en términos generales podemos decir que se basan en valorar los diversos componentes del paisaje que podemos clasificarlos en 3: descriptores físicos, artísticos y psicológicos (Gobster, H.P. and Chenoweth, R.E. 1989); no obstante, para facilitar el manejo podemos agruparlos en dos: descriptores físicos y descriptores-estéticos que incluirían los artísticos y los psicológicos (Cañas, I. 1992). 


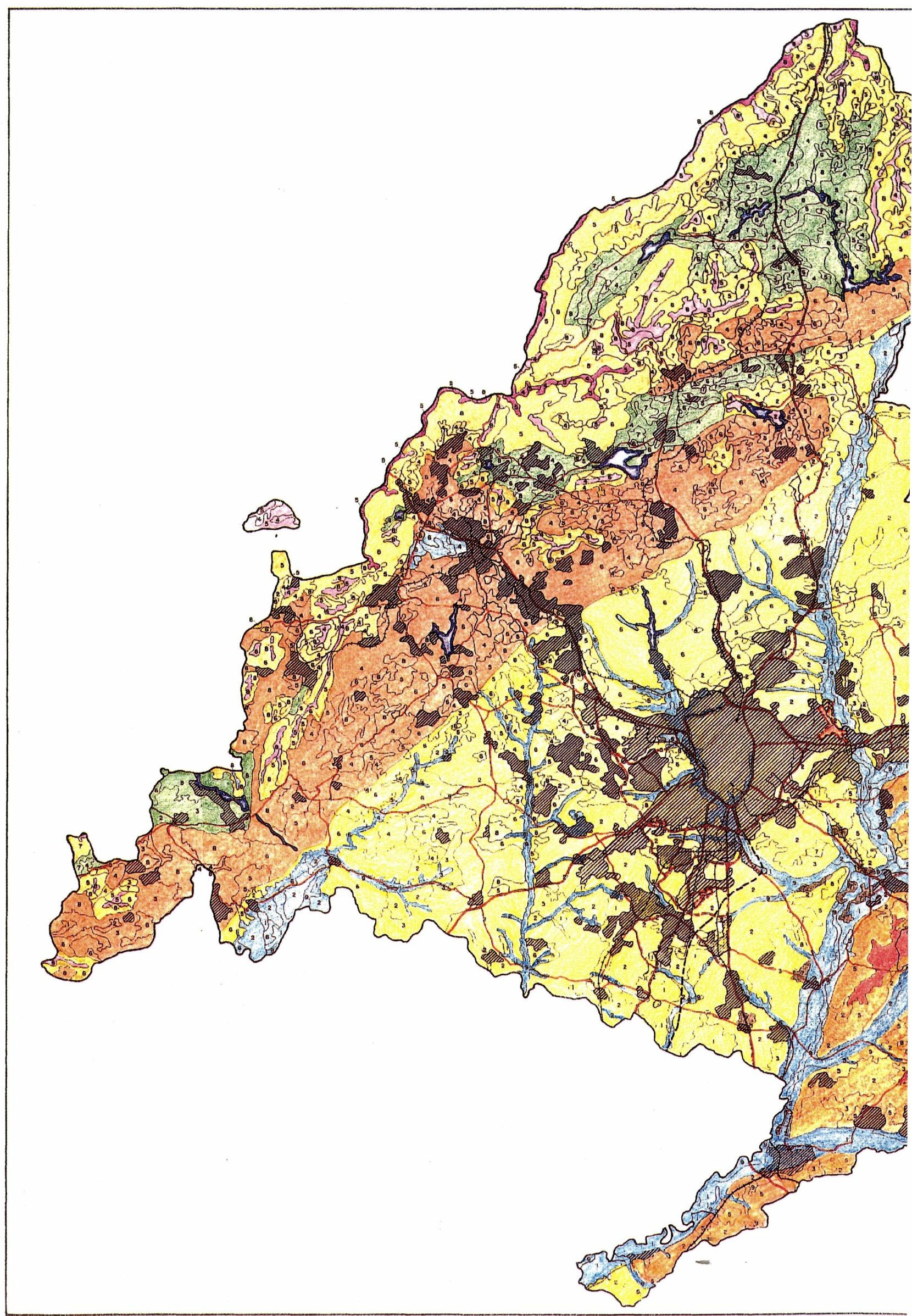




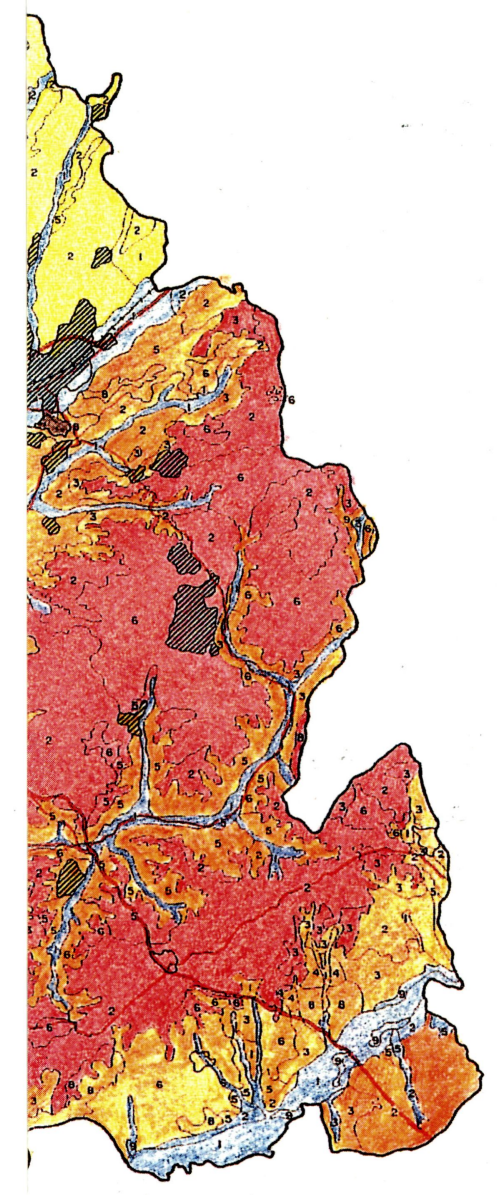

\section{LEYENDA}

UNIDADES FISIOGRÁFICAS

LA SIERRA

MUAR CUMBRES CIMERAS

$\square$ PARAMERAS SERRANAS

$\square$ LADERAS

AW VALLES INTERIORES

RAMPA

LA DEPRESIÓN
PÁRAMOS
TALUDES'DE PÁRAMO
CAMPIÑA
VEGAS FLUVIALES

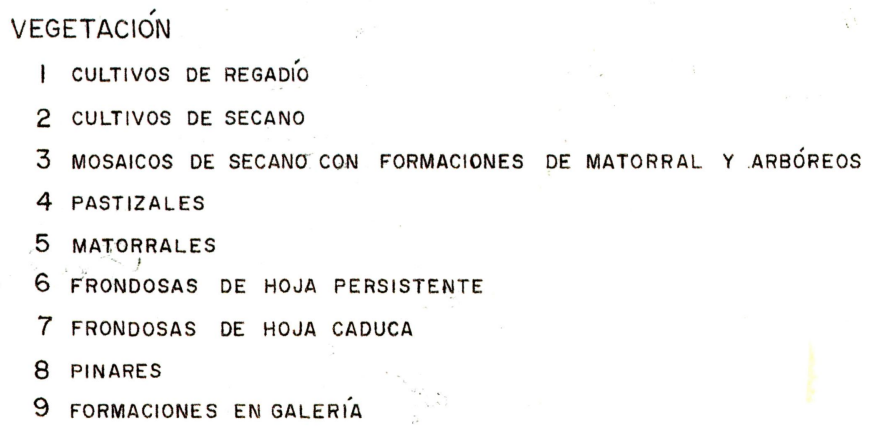

\section{MODIFICACIONES ANTRÓPICAS}

ZONAS AFECTADAS POR LA EXPANSIÓN URBANA


ZONAS AFECTADAS POR LA RED DE TRANSPORTES:

AEROPUERTO
AUTOPISTA, AUTOVIAA
CARRETERA
FERROCARRIL
FERROCARRIL ALTA VELOCIDAD
AUTOPISTA, AUTOVIÁ EN CONSTRUCCIÓN
OEN PROYECTO

EMBALSES O EN PROYECTO

Mapa 1.- Ejemplo de paisajes regionales, en este caso de la Comunidad de Madrid.

\section{PAISAJES REGIONALES}

COMUNIDAD AUTONOMA DE MADRID 


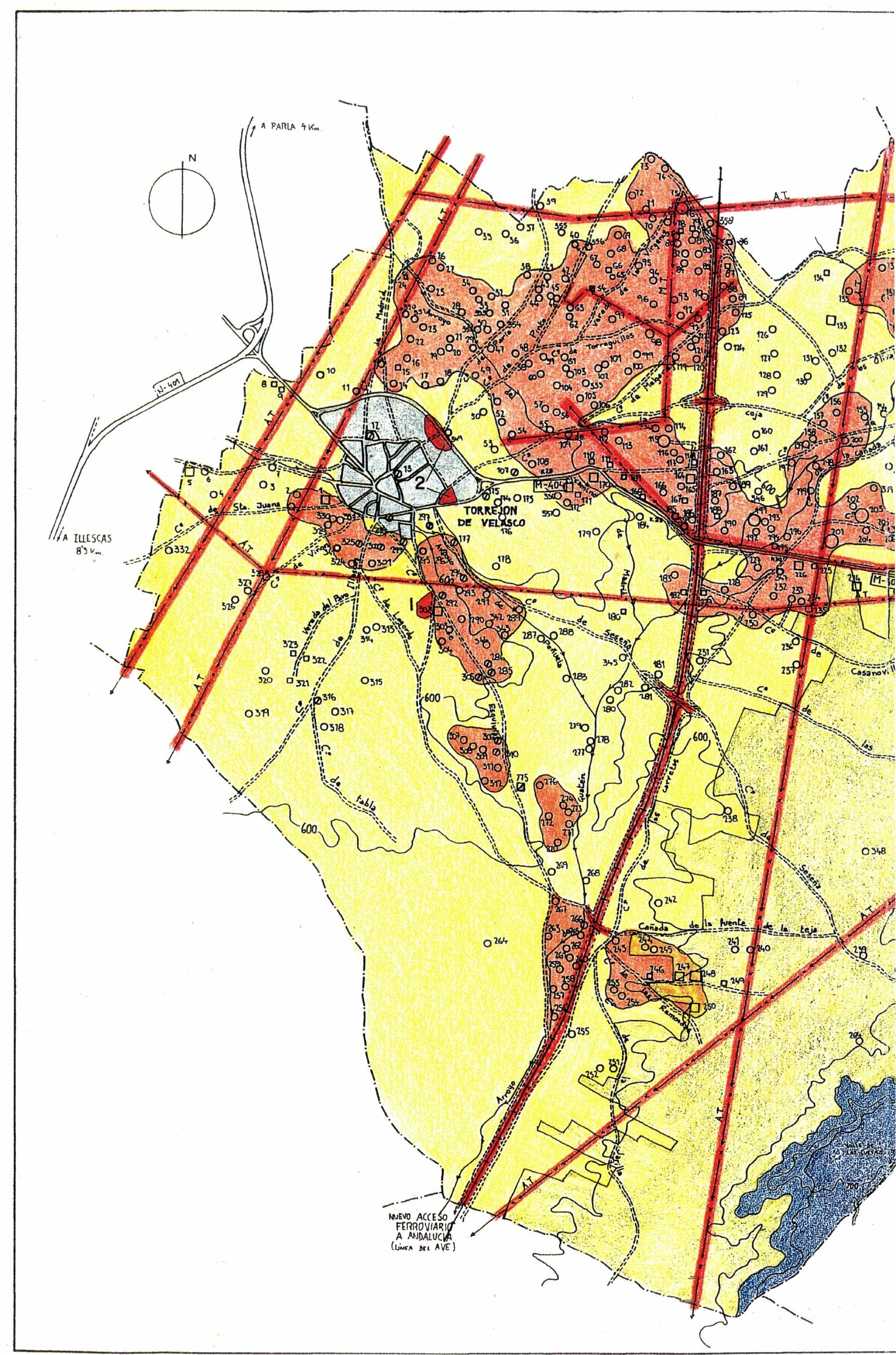




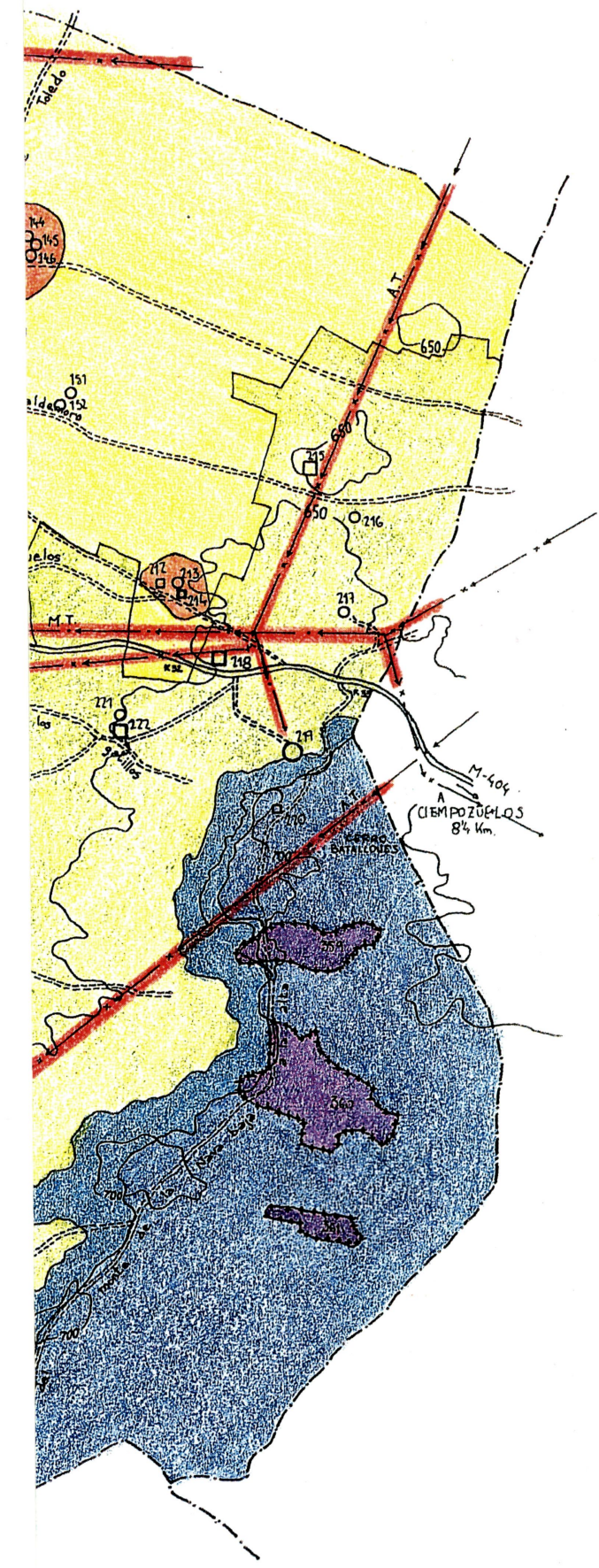

\begin{tabular}{lcll} 
GRUPO DE PAISAJE & DENOMINACIÓN & TIPO \\
\hline DEGRADADO (I'5 ptos.) & PAISAJE & DE CANTERAS & I-D \\
DEGRADADO (14 ptos.) & $"$ & SECANO DEGRADADO & $11-\mathrm{D}$ \\
DEFICIENTE (29'5 ptos.) & $"$ & SECANO & 1 \\
MEDIOCRE (32'5 ptos.) & $"$ & PLATAFORMA & 11 \\
MEDIOCRE (34 ptos.) & $"$ & OLIVOS CON INSTALACIONES & $111-\mathrm{D}$ \\
BUENO-MEDIO (44 ptos.) & $"$ & OLIVOS & III \\
BUENO-MEDIO (44 ptos.) & $"$ & CORNISA & IV
\end{tabular}

FOCOS DE DEGRADACIÓN VISUAL

ESTRUCTURAS LINEALES
ESTRUCTURAS PUNTUALES :
DESMONTE Y BASURERO
EXCESIVAS ESTRUCTURAS
NúCLEO URBANO

- VIVIENDA, INSTALACIONES CON OTROS USOS

口 CONJUNTO DE VIVIENDAS O INSTALACIONES CON OTROS USOS

$\square$ RUINAS

- INSTALACIONES aUXILIARES Agrarias

- instalaciones agrarias

O GONJUNTO DE INSTALACIONES AGRARIAS

[D] INVERnADEROS

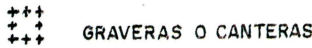

Mapa 2.- Paisajes de Torrejón de Velasco.

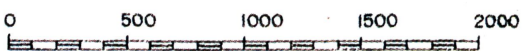


Como ejemplo de descriptores físicos tenemos: forma del terreno, vegetación, nieve, agua, etc.; de descriptores artísticos tendríamos: forma, color, textura; y descriptores psicológicos serían: afectividad, estimulación, simbolismo.

En el mapa 2 aparece un ejemplo de diversos paisajes, su delimitación y valoración. La valoración se hace en el campo una vez estudiados los límites de diversos descriptores físicos y estéticos a partir de la fotografía aérea. Según el sistema de evaluación de paisajes se da a cada descriptor una puntuación, sumándose después los puntos dando al final la valoración total del paisaje. Esto se realiza comparando las alternativas antes y después de la realización de la carretera; la diferencia en puntos es la pérdida de valor paisajístico del área, debida al desarrollo del proyecto.

\subsection{Valoración del proyecto}

Una de las variables que más influye en la integración en el paisaje de una vía es su adaptación al relieve atravesado; en términos generales deberá conservar las líneas estructurales o "carácter" del tipo de terreno en el que se sitúa.

En cuanto a la sección transversal, se ve influenciada también por numerosas variables como son: número de carriles, anchura y separación entre calzadas. Así se establecen relaciones de oposición distintas, si por ejemplo una carretera de cuatro carriles cuenta o no con mediana. La anchura de los carriles ayuda a determinar la escala visual y la dominancia de la carretera. Los arcenes, las aceras y los márgenes de la carretera juegan un papel igualmente importante en este sentido. Mención aparte merece el tratamiento de taludes en donde llama la atención la inclinación de éstos, el color, las plantaciones y el estado de conservación.

Será necesario tener en cuenta las diversas estructuras, instalaciones, señales, sistemas de control de tráfico, etc., para una correcta integración; normalmente no deberán ser dominantes a no ser que sea por conservar ciertos motivos propios de la zona.

Para la valoración del proyecto lo más útil es elaborar una lista de chequeo de los diferentes elementos y darle una puntuación según el diseño que podrá tomar valores positivos o negativos. Algunos autores ( Cats-Baril and Gibson 1987) utilizan ciertos parámetros para valorar el diseño como son: autenticidad, composición, fascinación etc; que pueden ser útiles cuando se desea un análisis fino.

\subsection{Estudio de los observadores}

\subsubsection{Facilidad de apreciarse}

La facilidad de verse o apreciarse la carretera depende de las características de la propia carretera, del paisaje y de la situación del observador. Cuando se requiere un estudio con cierto detalle se suele elaborar las áreas vistas, es decir, la superficie del territorio que se puede ver desde la carretera. Este mapa se superpone con el de paisajes indicando cuáles son los que se ven desde la vía.

Si se quisiera comparar alternativas, una forma sencilla sería multiplicando los kilómetros de carretera por el valor de cada paisaje que se ve. Así, por ejemplo, si tenemos dos alternativas de trazado:

- Alternativa 1: Paisaje A con 30 puntos y $7 \mathrm{~km}$ de carretera. Paisaje B con 45 puntos y $3 \mathrm{~km}$ de carretera. Paisaje $\mathrm{C}$ con 55 puntos y $2 \mathrm{~km}$ de carretera.

- Alternativa 2: Paisaje D con 35 puntos y $10 \mathrm{~km}$ de carretera. Paisaje E con 40 puntos y $2 \mathrm{~km}$ de carretera.

Valoración de la alternativa 1: $30 \times 7+45 \times 3+55 \times 2=$ 455 puntos.

Valoración de la alternativa $2: 35 \times 10+40 \times 2=430$ puntos.

Es decir que escogeríamos a igualdad de otros factores la alternativa 2 .

La comparación se podría hacer, también, por superficie e incluso especificando la visión frontal y lateral del observador (que en general dependen de la velocidad) e incluso distinguiendo, dentro de cada una, diversas zonas del paisaje como hacen por ejemplo: Landscape Architecture Research Office (1968) o Burke (1968).

El trabajo puede complicarse al considerar con mayor detalle los factores físicos que condicionan la respuesta de los observadores, como son por ejemplo la distancia y posición de éstos.

A veces el estudio de las áreas vistas y la mapificación de éstas puede ayudar a una mayor aceptación del proyecto, ya que en algunos easos la población puede estar sensibilizada por la idea de que afectará a una gran zona del paisaje. 




Los taludes tienen, si no se tratan convenientemente, un efecto negativo en la integración de la vía en el paisaje. En algunos estudios se supone que en "la España húmeda" no es necesario el tratamiento de los taludes, pues se recubriria con vegetación de forma natural. En la fotografía se observa un tramo de la N-547 en la provincia de La Coruña.

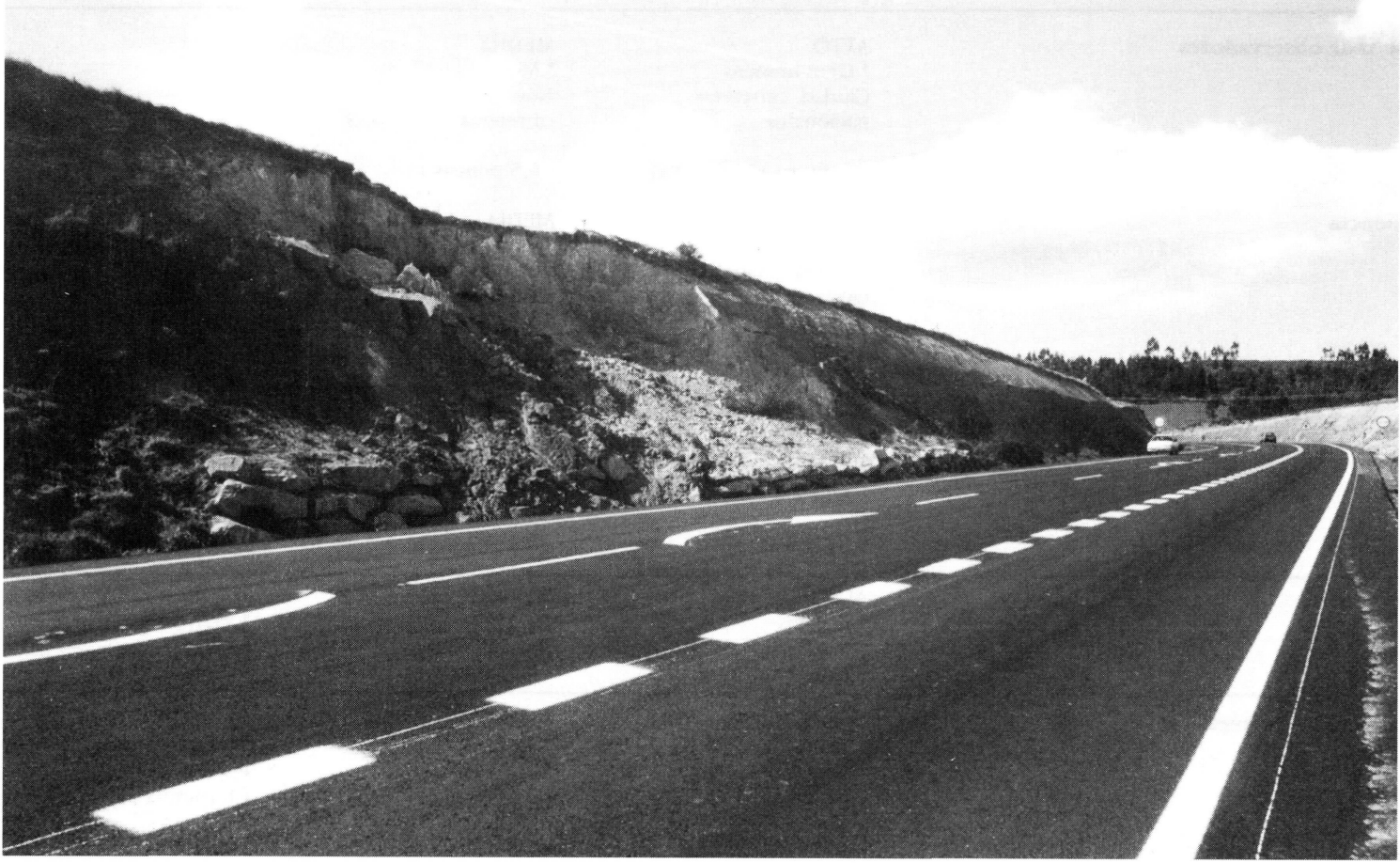

Además de las razones estéticas, el tratamiento correcto de taludes evita el desprendimiento de tierras sobre la vía. 


\subsubsection{Número de observadores}

Uno de los factores más importantes de la respuesta del observador es el conocimiento exacto del número de ellos, aunque lo más normal es estimar el número potencial de observadores afectados. Es también conveniente, en muchos casos, estimar los observadores desde la carretera frente a los observadores de la carretera, ya que éstos suelen tener en ocasiones intereses contrarios.

\subsubsection{Interés de los observadores}

En este epígrafe incluimos el interés o sensibilidad de los observadores, queriendo significar la receptibilidad de los diferentes grupos de observadores al entorno. Un nivel de sensibilidad alto del observador puede ser crítico para la planificación del proyecto, ya que un aumento de la respuesta del observador incrementa la importancia de los objetivos visuales llegando en algunos casos ante una alta sensibilidad del observador el recomendar que no se realice ningún cambio.
La receptibilidad del observador está relacionada con la preferencia visual y ésta se ve influenciada por la actividad del observador, su conocimiento, sus opiniones y prejuicios. Es claro que el interés que muestra un trabajador de una industria pesada por el entorno donde trabaja suele ser diferente del que está en una zona recreativa, en este sentido se pueden distinguir los observadores según la actividad observada en grandes grupos: observadores de zonas residenciales, observadores de zonas recreativas, observadores de zonas industriales y observadores de zonas de servicios.

El conocimiento del observador se refiere bien a la experiencia remota: sensibilidad hacia temas medioambientales, o bien a la experiencia inmediata. En esta segunda puede tener un papel importante el equipo que diseñe la carretera al jugar con el cambio o contraste visual. En general una transición de un paisaje a otro puede aumentar el conocimiento de los precedentes. Este contraste se da en grado extremo al entrar en una gran urbe, o el contraste de zona montañosa a llana ( por ejemplo el que se da en el puerto de Pajares entre León y Asturias).

Cuadro 1: Cuadro sencillo de respuesta del observador

\begin{tabular}{|c|c|c|c|}
\hline \multirow[t]{2}{*}{ Número de observadores } & $\begin{array}{l}\text { ALTO } \\
\text { "Gran número } \\
\text { Ciudad, carreteras } \\
\text { nacionales }\end{array}$ & $\begin{array}{l}\text { MEDIO } \\
\text { * Medio } \\
\text { Núcleos rurales } \\
\text { carreteras comarcales }\end{array}$ & $\begin{array}{l}\text { BAJO } \\
\text { * Pocos }\end{array}$ \\
\hline & ( 1 punto ) & ( 0,5 puntos $)$ & ( 0 puntos) \\
\hline \multirow[t]{2}{*}{ Frecuencia } & $\begin{array}{l}\text { ALTA } \\
\text { * Muy frecuente } \\
\text { Zona de paso entre } \\
\text { ciudades o núcleos con } \\
\text { más de } 50.000 \text { habitantes }\end{array}$ & $\begin{array}{l}\text { MEDIA } \\
\text { * Ocasionales } \\
\text { Zona de paso entre } \\
\text { núcleos con más de } \\
10.000 \text { habitantes }\end{array}$ & $\begin{array}{l}\text { BAJA } \\
{ }^{*} \text { Infrecuentes }\end{array}$ \\
\hline & ( 1 punto ) & ( 0,5 puntos $)$ & ( 0 puntos ) \\
\hline \multirow[t]{2}{*}{ Duración } & $\begin{array}{l}\text { ALTA } \\
\text { "Largo tiempo } \\
\text { Zonas con } \\
\text { miradores, plazas }\end{array}$ & $\begin{array}{l}\text { MEDIA } \\
{ }^{*} \text { Intermedio } \\
\text { Zonas estacionamiento }\end{array}$ & $\begin{array}{l}\text { BAJA } \\
\text { * Muy poco tiempo }\end{array}$ \\
\hline & ( 1 punto ) & $(0,5$ puntos $)$ & ( 0 puntos ) \\
\hline Interés & $\begin{array}{l}\text { ALTO } \\
\text { * Turistas } \\
\text { (Interés nacional) } \\
(1 \text { punto ) }\end{array}$ & $\begin{array}{l}\text { MEDIO } \\
\text { * Turistas } \\
\text { (Interés regional) } \\
\text { ( } 0,5 \text { puntos ) }\end{array}$ & $\begin{array}{l}\text { BAJO } \\
\text { *Transeúntes (no } \\
\text { turistas) } \\
\text { ( } 0 \text { puntos ) }\end{array}$ \\
\hline Localización carretera & $\begin{array}{l}\text { MUY ELEVADA } \\
\text { * Muy elevado } \\
>20 \mathrm{~m} \\
\text { ( } 1 \text { punto ) }\end{array}$ & $\begin{array}{l}\text { ELEVADA } \\
\text { * Elevado } \\
5-20 \mathrm{~m} \\
(0,5 \text { puntos })\end{array}$ & $\begin{array}{l}\text { AL NIVEL } \\
* \text { Al nivel del } \\
\text { terreno } \\
(0 \text { puntos })\end{array}$ \\
\hline $\begin{array}{l}\text { Posición del observador } \\
\text { ( Se observa descle ) }\end{array}$ & $\begin{array}{l}\text { IMPORTANTE } \\
\text { * Escuelas, hospitales } \\
\text { áreas recreativas, } \\
\text { autovías, carreteras } \\
\text { nacionales. }\end{array}$ & $\begin{array}{l}\text { NORMAL } \\
\text { *Áreas comunes, } \\
\text { carreteras. }\end{array}$ & $\begin{array}{l}\text { BAJA } \\
{ }^{*} \text { Tierras de } \\
\text { cultivo, áreas } \\
\text { industriales, } \\
\text { caminơs secundararios }\end{array}$ \\
\hline & ( 1 punto) & $(0,5$ puntos $)$ & ( 0 puntos ) \\
\hline
\end{tabular}


Cuadro 2: Establecimiento de clases en la adecuación de la carretera al paisaje en función de los puntos

\begin{tabular}{|lcc|}
\hline & & \\
BUENA & $>+5$ & Puntos \\
BUENA-REGULAR & $+5 \mathrm{a}-5$ & Puntos \\
REGULAR & $-5 \mathrm{a}-15$ & Puntos \\
REGULAR-MALA & $-15 \mathrm{a}-30$ & Puntos \\
MALA & $<-30$ & Puntos \\
\hline
\end{tabular}

Cuadro 3: Clasificación global de impacto de una carretera en el paisaje en función de la adaptación de la carretera al paisaje y la puntuación de éste.

\begin{tabular}{|l|llllll|}
\hline & ADECUACIÓN & Mala & Regular-Mal & Regular & Buena-Reg. & Buena \\
\cline { 2 - 4 } & Dudoso & Mejorable & Admisible & Correcto & Correcto \\
DEGRADADO & Inadmisible & Dudoso & Admisible & Correcto & Correcto \\
DEFICIENTE & Inadmisible & Dudoso & Mejorable & Admisible & Correcto \\
MEDIOCRE & Inadmisible & Inadmisible & Dudoso & Mejorable & Admisible \\
BUENA & Inadmisible & Inadmisible & Inadmisible & Dudoso & Mejorable \\
NOTABLE & Inadmisible & Inadmisible & Inadmisible & Inadmisible & Mejorable \\
MUY BUENA & Inadmisible & Inadmisible & Inadmisible & Inadmisible & Dudoso \\
\hline
\end{tabular}

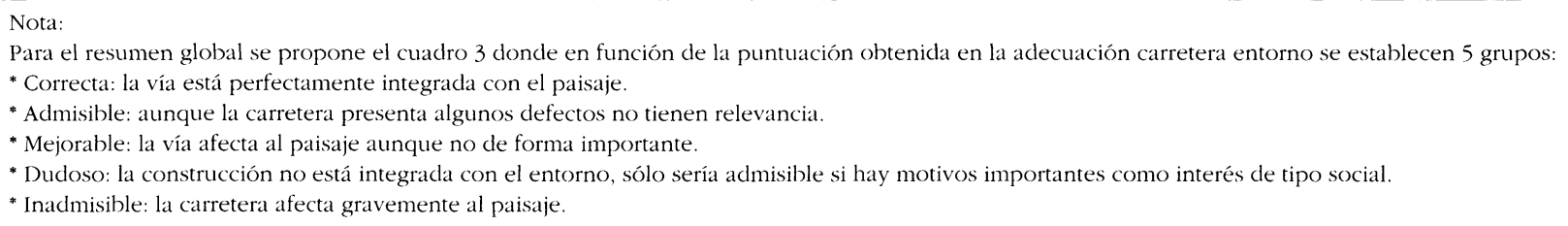

Las opiniones y prejuicios actúan indirectamente sobre la experiencia del observador, desarrollando expectaciones y apreciaciones. Los observadores pueden ser particularmente sensibles a algunos recursos con un cierto significado tradicional que desde otros puntos de vista pueden tener poco valor.

Si el estudio no tiene especiales problemas puede utilizarse un sistema sencillo, como el que aparece en el cuadro 1 , donde se valora la respuesta del observador.

\subsection{Valoración global}

En la última parte del estudio del impacto paisajístico se trata de integrar la adaptación de la carretera al paisaje con la respuesta del observador. Un método sencillo consiste en hacer 5 grupos de adecuación y según los puntos obtenidos incluirlo en una de ellas.
Estas categorías se cruzan con la valoración del paisaje dando el valor del impacto. En el cuadro 2 y 3 aparecen unos ejemplos de cuadros de adaptación y de valoración del impacto.

\section{BIBLIOGRAFÍA}

BURKE, H.D. et al. 1968. "A Method for Classifing Scenery from a Roadway ". Park Practice Guideline Development. Págs 125-140.

BLAIR, W.G.E.; ISAACSON, L. and JONES, G.R. 1979. "Da Comprehensive Approach to Visual Resource Management for Highway Agencies". In Proceedings of Our National Landscape. A Conference on Applied Techniques for Analysis and Management of the Visual Resource. U.S.D.A. Forest. Berkeley. Págs 395-373. 
CAÑAS, I. 1992. Integración de las construcciones agrarias en el paisaje: el color: Tesis doctoral presentada en la Universidad Politécnica de Madrid. Sin publicar.

CHAPLIN, B.L. 1979. " Highway Development " In Proceedings of Our National Landscape. A Conference on Applied Techniques for Analysis and Management of the Visual Resource. U.S.D.A. Forest. Berkeley. Págs 44-47.

GOBSTER, P.H. and CHENOWETH, R.E. 1989. "The Dimensions of Aesthetic Preference: a Quantitative Analysis". Journal of Environmental Management. 29, págs 47-72.

FERNANDEZ DEL CAMPO, J.A. 1983. Factores estéticos y ecológicos en el trazado. Simposium sobre trazado de carreteras. A. E. C. Barcelona.

HAMPE, G.D. and NOE, F.P. 1979. "Highway Attitudes and Levels of Roadside Maintenance ". In Proceedings of Our National Landscape. A Conference on Applied Techniques for Analysis and Management of the Visual Resource. U.S.ID.A. Forest. Berkeley. Págs 373-379

HULL, R.B. ; BUHYOFF G.J ; and CORDELL H.K. 1987. " Psychophysical Models: An Example with Scenic Beauty Perceptions of Roadside Pine Forests "Landscape Joumal. V6, n 2, págs 113-122.
LANDSCAPE ARCHITECTURE RESEARCH OFFICE. 1968. Highway Esthetics Functional Criteria for Planning and Design. Harvard University, Graduate School of Design.

McCOOL, S.F. 1979. "The Off-Road Recreation Vehicle - Visual Impacts ". In Proceedings of Our National Landscape. A Conference on Applied Techniques for Analysis and Management of the Visual Resource. U.S.D.A. Forest. Berkeley. Págs 73-76.

NATIONAL FOREST LANDSCAPE MANAGEMENT. 1977. Roads. V 2, Chapter 4. Forest Service, U.S. Department of Agriculture, March 1977.

PEREZ OLOZAGA, C. 1992. La evaluación de impacto ambiental de la Autovía que une Navarra con la CN-I en Aduna (Tramo de Guipúzcoa). Transporte y medioambiente. MOPT. Madrid.

POTTER, R.I. and WAGAR, J.A. 1971. Techniques for Inventorying Manmade Impacts In Roadway Environments. Pacific Northwest Forest and Range Experiment Station Forest Service. U.S. Department of Agriculture. Portland, Oregon.

SHANAHAN D. et al. 1986. Preserving New York State Scenic roads. A Guide to Designation. New York State.

U.S.D.O.T. 1981. Visual Impact Assessmente for Higway Proyects. Federal Highway Administration Office of Environmental Policy. 